\title{
The effect of thermal annealing on dopant site choice in conjugated polymers
}

\author{
Jun Li ${ }^{\mathrm{a}}$, Christopher W. Rochester ${ }^{\mathrm{a}}$, Ian E. Jacobs ${ }^{\mathrm{a}}$, Erik W. Aasen ${ }^{\mathrm{a}}$, Stephan Friedrich ${ }^{\mathrm{b}}$, Pieter Stroeve ${ }^{\mathrm{a}}$, Adam J. Mouléa,* \\ ${ }^{a}$ Chemical Engineering and Materials Science, University of California, Davis, CA 95616, USA \\ ${ }^{b}$ Advanced Detector Group, Lawrence Livermore National Laboratory, Livermore, CA 94550, USA
}

\begin{abstract}
Solution-processed organic electronic devices often consist of layers of polar and non-polar polymers. In addition, either of these layers could be doped with small molecular dopants. It is extremely important for device stability to understand the diffusion behavior of these molecular dopants under the thermal stress and whether the dopants have preference for the polar or the non-polar polymer layers. In this work, a widely used molecular dopant 2,3,5,6-tetrafluoro-7,7,8,8-tetracyanoquinodimethane (F4TCNQ) was chosen to investigate dopant site preference upon thermal annealing between the polar thiophene poly(thiophene-3-[2-(2methoxy-ethoxy)ethoxy]-2,5-diyl) (S-P3MEET) and non-polar thiophene poly(3-hexylthiophene) (P3HT). F4TCNQ is able to ptype dope both P3HT and S-P3MEET. Further doping studies of S-P3MEET using near edge X-ray absorption fine structure spectroscopy, conductivity measurements and atomic force microscopy show that the F4TCNQ additive competes for doping sites with the covalently attached dopants on the S-P3MEET. Calorimetry measurements reveal that the F4TCNQ interacts strongly with the side-chains of the S-P3MEET, increasing the melting temperature of the side-chains by $30{ }^{\circ} \mathrm{C}$ with $5 \mathrm{wt} \%$ dopant loading. Next, the thermal stability of doping in the polar/non-polar (S-P3MEET/P3HT) bilayer architectures was investigated. Steadystate absorbance and fluorescence results show that F4TCNQ binds much more strongly in S-P3MEET than P3HT and very little F4TCNQ is found in the P3HT layer after annealing. In combination with reflectometry measurements, we show that F4TCNQ remains in the SP3MEET layer with annealing to $210{ }^{\circ} \mathrm{C}$ even though the sublimation temperature for neat F4TCNQ is about 80 ${ }^{\circ} \mathrm{C}$. In contrast, F4TCNQ slowly diffuses out of P3HT at room temperature. We attribute this difference in binding the F4TCNQ anion to the ability of the ethyl-oxy side-chains of the S-P3MEET to orient around the charged dopant molecule and thereby to stabilize its position. This study suggests that polar side-chains could be engineered to increase the thermal stability of molecular dopant.
\end{abstract}

Keywords:

Organic electronics; Molecular doping; Molecular diffusion; Thermal stability

\section{Introduction}

Organic electronic materials and devices have recently been extensively studied because they show the potential to replace inorganic components for some applications, including organic photovoltaics (OPVs) and organic light emitting diodes (OLEDs) [1, 2, 3]. Organic electronic devices often consist of multiple thin (5-100 nm) layers, each of which has a specific electronic and/or optical function. It is tempting to assume that the device will operate as if each layer is discrete, pure, and has sharp interfaces. In practice, however, it is difficult to control the composition and morphology of these layered materials because of chemical reactions at layer interfaces and diffusion of mobile species through the interface $[4,5]$. Since the thermal stability of the layers and interfaces are essential in determining the quality and stability of the devices, the characterization of organic layer properties in connection with thermal stress is imperative in understanding the mechanisms involved in device degradation $[6,7]$.

${ }^{*}$ Corresponding author, Phone: +01 530 754-8669, Fax: +01 530 752-1074

Email address: amoule@ucdavis . edu (Adam J. Moulé)
Many solution-processed organic electronic devices incorporate a polar (hydrophilic) layer and a non-polar (hydrophobic) layer because the use of orthogonal solvents allows layer

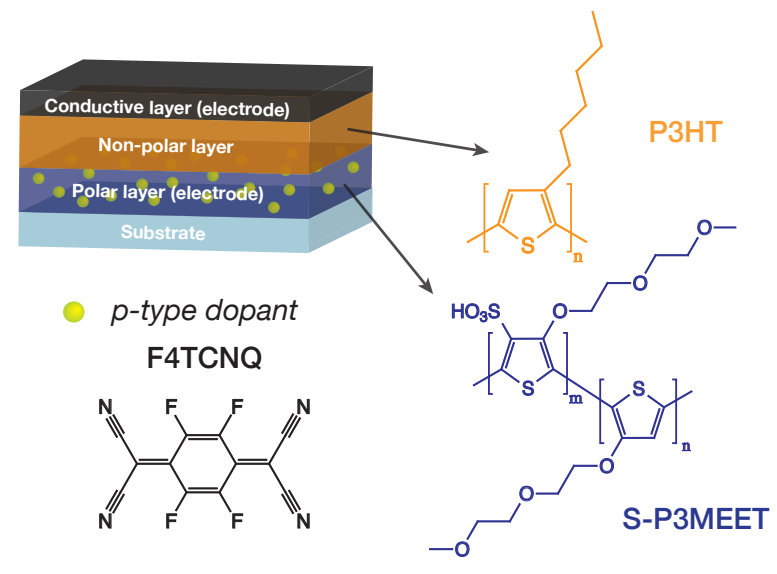

Figure 1: Schematic diagram of a typical solution-processed device structure (upper left) and molecular structures of P3HT (upper right), S-P3MEET (bottom right) and F4TCNQ (bottom left). 
stacking (Fig. 1). In fact, the most widely used material in solution-processed organic electronic devices is the transparent conductive polymer poly(3,4-ethylenedioxythiophene)poly(styrenesulfonate) (PEDOT:PSS) because of this layering ability $[8,9,10,11]$. However, even covalently bound ionic dopants have been shown to cause inadvertent doping of adjacent layers. Excess polymeric dopant (PSS) has a tendency to phase segregate into a surface layer on top of the PEDOT:PSS mixture, and after heat treatment mix with and dope the adjacent non-polar semiconducting polymer layer [12, 13, 14, 15]. A similar effect has also been observed in mixtures of another polymeric dopant, perfluorinated ionomer (PFI), with poly(thiophene-3-[2-(2-methoxyethoxy)ethoxy]-2,5-diyl) (S-P3MEET). It was shown that PFI tends to phase separate out of the polymer due to its low miscibility in conjugated polymers, forming a PFI-rich skin between S-P3MEET and poly(3-hexylthiophene) (P3HT) layers. In this case, the location of the dopant remains stable under considerable thermal stress, which implies that reduced mixing can hinder undesired doping [16]. This relationship between ionic dopant miscibility and contact doping across a polar/non-polar interface was recently quantified for a variety of self-doped polymers [17].

By comparison, molecular dopants are more traditionally used in small molecule evaporated devices. Since the dopant is a separate molecule, it can be co-evaporated with a hole or electron transport material and causes doping by forming charge transfer salts in-situ in specific layers [18, 19]. This dopant type is used to tune the work function and/or induce selective n-type or p-type charge transport [20]. A disadvantage, however, is that these dopants are not covalently bound into position and thereby can diffuse into other layers within the device under thermal or electronic stress. The weaker van der Waals or Coulombic forces at play in these samples is not sufficient to bind the dopant into place $[21,22]$. Li et al. recently showed that 2,3,5,6-tetrafluoro7,7,8,8-tetracyanoquinodimethane (F4TCNQ) sublimates out of the N,N,N',N'-tetrakis(4-methoxy-phenyl)benzidine (MeOTPD) mixed layer at $\sim 150{ }^{\circ} \mathrm{C}$ and diffuses at room temperature [23]. F4TCNQ has also been investigated as a p-type dopant for polymer samples using solution-processing with many of these studies focused on the non-polar polymer P3HT [24, 25, 26]. More recently, F4TCNQ was used to control the solubility of P3HT for patterning purposes and is likely therefore to be incorporated into solution-processed devices in the near future [27]. Unfortunately, little attention has been paid to the question of whether the molecular dopants have preference for the polar layer or for the non-polar layer, which is of high interest from the device stability perspective.

In this study, conjugated polymers, S-P3MEET and P3HT (Fig. 1), will be used to fabricate the polar and non-polar layers, respectively. S-P3MEET and P3HT are chosen because 1) both materials have similar polythiophene backbones; 2) P3HT is the most widely used conjugated polymer while S-P3MEET has been shown as a promising alternative to PEDOT:PSS [28, 16], and 3) S-P3MEET forms a thermally stable bilayer with P3HT, so we can study the transport of F4TCNQ without interfer- ence from mixing between the layers. The first part of this paper explores whether F4TCNQ is able to dope S-P3MEET. A comprehensive study on the electronic, morphological and thermal properties of the F4TCNQ doped S-P3MEET layer is performed. Next, a combination of absorbance/fluorescence spectroscopy and X-ray reflectometry techniques are used to investigate the location of F4TCNQ upon thermal annealing of bilayers of polar S-P3MEET and non-polar P3HT.

\section{Materials and Methods}

\subsection{Materials and Sample Preparation}

The S-P3MEET formulation (Plexcore OC RG-1100) was provided by Plextronics, inc. F4TCNQ ( $\geq 97 \%)$, Poly(3hexylthiophene-2,5-diyl) and (P3HT) (Regioregular, $M_{n}=54-$ $75 \mathrm{kDa}$ ) were purchased from Sigma-Aldrich. PEDOT:PSS (Clevios P VP 4083) was purchased from Heraeus. All chemicals were used as-received unless otherwise indicated.

For sample preparation, all substrates were cleaned using ultrasonic baths of acetone, 5\% Mucasol solution, and deionized water, successively, followed by nitrogen drying. The substrates were then placed to a UV-ozone cleaner for $30 \mathrm{~min}$ before use. The F4TCNQ solution used to dope the S-P3MEET was prepared by dissolving F4TCNQ in a solvent mixture that consisted of 50\% isopropyl alcohol, 40\% 1,3-propanediol, and $10 \%$ deionized water by volume to a concentration of $1 \mathrm{mg} / \mathrm{ml}$. Solutions of S-P3MEET and F4TCNQ were then mixed in appropriate ratios to achieve the desired doping levels. The films were then spin-coated on clean substrates from blend solutions, and placed on a hotplate at $110{ }^{\circ} \mathrm{C}$ for 3 minutes to remove excess solvent.

\subsection{Characterization}

Steady-state absorbance and photoluminescence spectra were recorded on a Perkin Elmer Lambda 750 spectrophotometer and a Varian Eclipse photoluminescence spectrophotometer, respectively. The photoluminescence experiments were conducted using a $2.38 \mathrm{eV}$ excitation beam to optically excite the P3HT. Spectroscopy measurements were performed on P3HT/F4TCNQ bilayer samples. For one of the P3HT/F4TCNQ bilayer samples, the P3HT was first spincoated onto a glass substrate to form an $80 \mathrm{~nm}$ thick film. The sample was then placed in a MBraun thermal evaporator, which was pumped down to a pressure of $5 \times 10^{-6}$ mbar before depositing $10 \mathrm{~nm}$ of F4TCNQ onto the P3HT film at a rate of $\sim 0.2$ $\AA /$ sec. Another P3HT/F4TCNQ bilayer was prepared by mechanically laminating the two individual layers together. This was accomplished by depositing the P3HT and F4TCNQ layers separately onto glass substrates and clamping the layers together with a force of approximately $0.3 \mathrm{~N} / \mathrm{cm}^{2}$. The two layers remained in contact for three weeks before the spectroscopy measurements were performed. For the S-P3MEET/P3HT bilayer sample, $60 \mathrm{~nm}$ P3HT layer was spin-coated on top of 60 $\mathrm{nm}$ undoped or doped S-P3MEET layer. The samples were measured before and after annealing. Last, the inverse P3HT/SP3MEET bilayer sample was also prepared by spin-coating 60 nm S-P3MEET onto $60 \mathrm{~nm}$ F4TCNQ doped P3HT film. 
Near edge X-ray absorption fine structure measurements were performed on beamline 6.3.1 at the Advanced Light Source (ALS) at Lawrence Berkeley National Laboratory (LBNL). Thin films of S-P3MEET and F4TCNQ doped SP3MEET were spin-coated onto ITO-coated glass substrates, and then heated at elevated temperatures for $5 \mathrm{~min}$ before the measurements. Carbon K-edge spectra were obtained by total electron yield (TEY) at a $\sim 30^{\circ}$ incidence angle using a Galileo 4716 channeltron electron multiplier, and the TEY signal was normalized by the incident beam intensity $\mathrm{I}_{0}$. The data were processed and analyzed using Athens software, where a linear background was subtracted, and the spectra were normalized above the edge at $320 \mathrm{eV}$.

For in-plane conductivity measurements, four electrodes (5 $\mathrm{nm} \mathrm{Cr} / 95 \mathrm{~nm} \mathrm{Au}, 1 \times 5 \mathrm{~mm}^{2}, 1 \mathrm{~mm}$ spacing) were deposited on clean glass substrates by thermal evaporation. Films were then spin-coated and measured with a four-point probe setup using a Keithely 2420 sourcemeter unit. All conductivity measurements were performed in the dark under a nitrogen atmosphere in a glovebox.

Atomic force microscopy measurements were performed in tapping mode using a Veeco Multimode microscope. $\sim 30 \mathrm{~nm}$ thickness films were prepared at each doping concentration. Silicon probes (Tap300Al-G) with a resonant frequency of 300 $\mathrm{kHz}$ and a force constant of $40 \mathrm{~N} / \mathrm{m}$ were used for imaging. Images were analyzed and processed using Veeco NanoScope Analysis software.

Differential scanning calorimetry measurements were performed using a Mettler-Toledo differential scanning calorimeter. The S-P3MEET and blend solutions were first drop-casted onto clean $1 \times 3$ in. $^{2}$ glass slides. The samples were then placed into a vacuum chamber for $30 \mathrm{~min}$, and placed on a hot plate in a nitrogen filled glovebox set to $110^{\circ} \mathrm{C}$ for $1 \mathrm{~min}$ to remove the residual solvent. The samples were then scraped from the glass slides using a clean razor blade. For the measurement, 5$6 \mathrm{mg}$ of the polymer samples were sealed into $40 \mu \mathrm{l}$ aluminum pans. The scans were taken with a temperature ramp rate of 10 ${ }^{\circ} \mathrm{C} / \mathrm{min}$ from 0 to $300{ }^{\circ} \mathrm{C}$.

X-ray reflectometry measurements were performed on beamline 2-1 at the Stanford Synchrotron Radiation Lightsource (SSRL). Silicon wafers (3 in. diameter) were used as substrates. The sample was prepared by first spin-coating a mixture of SP3MEET with F4TCNQ onto the substrate to obtain a film with thickness $\sim 30 \mathrm{~nm}$, and heated at $110{ }^{\circ} \mathrm{C}$ for $3 \mathrm{~min}$ to remove excess solvent. A $20 \mathrm{~nm}$ P3HT layer was then deposited on top. The measurements were performed using a $8 \mathrm{keV}$ incident beam in a He atmosphere and the reflectivity spectra were obtained using a Bicron scintillation point detector. The same sample was heated on a hot plate at elevated temperatures for 5 min and was remeasured after each heating step. The reflectivity data were analyzed using the MOTOFIT reflectometry analysis package within the IGOR software [29]. A SLAB model was used to represent the samples.

\section{Results and Discussion}

\subsection{Molecular Doping of the Non-Polar Layer}

P3HT has been extensively used in organic electronic devices. A considerable number of studies have already shown that the molecular dopant F4TCNQ is able to p-type dope P3HT [30, 24, 26, 31]. In this section, this molecular doping process is re-examined from the dopant diffusion perspective.

Instead of solution doped blend films seen in the literature, here a P3HT/F4TCNQ mixed layer was prepared by evaporating $10 \mathrm{~nm}$ of F4TCNQ onto an $80 \mathrm{~nm}$ P3HT film (upper cartoon in Fig. 2). The resulting UV-vis absorbance and photoluminescence (PL) spectra are shown in Fig. 2 (green dots). It is clear that P3HT and F4TCNQ do not form a stable bilayer structure, but rather the P3HT is substantially doped by the F4TCNQ molecules, as seen by the reduced P3HT absorbance around $2.48 \mathrm{eV}$ and the appearance of the broad peaks centered around $1.5 \mathrm{eV}$ and $0.6 \mathrm{eV}$ which are characteristics of $\mathrm{P} 3 \mathrm{HT}^{+}$polarons $[24,25]$. Based on the ground-state/polaron peak ratio, we can determine a doping concentration of $\sim 11$ wt\% [31], which agrees nicely with the amount of F4TCNQ we deposited. This result suggests that $\mathrm{P} 3 \mathrm{HT}$ provides a good host for F4TCNQ and that when it is present, the F4TCNQ efficiently dopes the entire P3HT layer at room temperature with an unambiguous change in the optical absorbance. The PL of P3HT in the inset of Fig. 2 shows complete quenching by the presence of F4TCNQ. These results imply that F4TCNQ diffuses throughout the P3HT layer, causing complete mixing between these two materials with only the thermal activation of the $80{ }^{\circ} \mathrm{C}$ thermal source.

To obtain room temperature diffusion results, another P3HT/F4TCNQ bilayer sample was prepared by mechanically stacking neat layers of F4TCNQ on glass and P3HT on glass together for three weeks (lower cartoon in Fig. 2). Any change in the spectra is attributed to room temperature diffusion between separate films. The spectrum of the P3HT film after contact

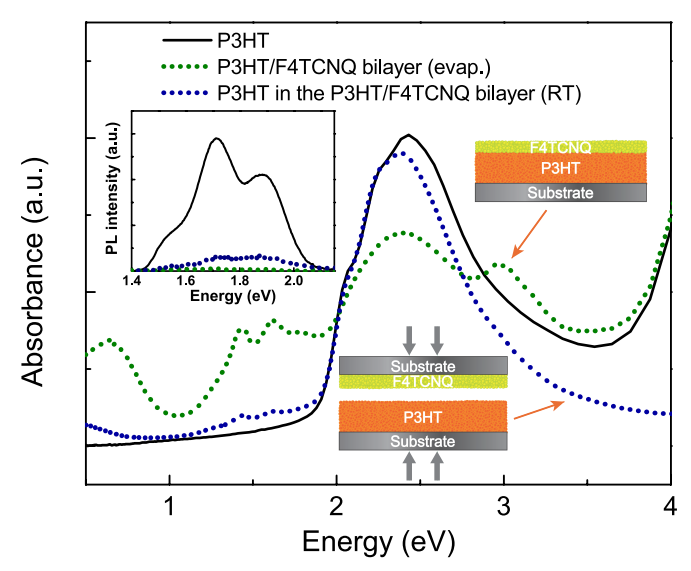

Figure 2: UV-vis-NIR absorbance spectra of a bilayer of sequentially thermal evaporating $10 \mathrm{~nm}$ of F4TCNQ on top of $80 \mathrm{~nm}$ P3HT film (green dots) and the $80 \mathrm{~nm}$ P3HT film after mechanically being laminated with $10 \mathrm{~nm}$ F4TCNQ at room temperature for three weeks (blue dots). Inset cartoon: prepared bilayer structure. Inset figure: photoluminescence spectra of these samples. 


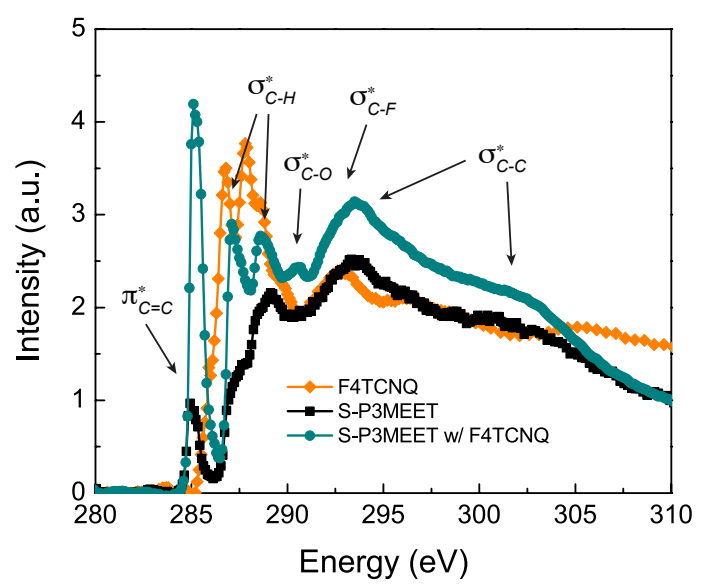

Figure 3: Carbon K-edge NEXAFS spectra of S-P3MEET, F4TCNQ and blend film of S-P3MEET with $1 \mathrm{wt} \%$ F4TCNQ. The peak assignments are also shown.

(blue dots) is also depicted in Fig. 2. It shows that the F4TCNQ does diffuse into the P3HT at room temperature by the appearance of the same doping features in the absorbance spectrum around $1.5 \mathrm{eV}$ and $0.6 \mathrm{eV}$. However, in this case, the $\mathrm{P}_{3} \mathrm{HT}^{+}$ polaron peaks are less pronounced and the PL quenching of the P3HT is reduced compared with the evaporated sample, indicating that F4TCNQ diffuses into the P3HT layer rather slowly at room temperature ( $2.2 \mathrm{wt} \%$ doping concentration).

\subsection{Molecular Doping of the Polar Layer}

It has been shown in the above section that F4TCNQ is able to p-type dope non-polar P3HT layer. Theoretically, such interaction is expected if the ionization energy (IE) of the polymer is lower than the electron affinity (EA) of p-type dopant. However, the IE and EA values reported from different groups vary to a large extent, e.g. the $\mathrm{IE}_{P 3 H T}$ was reported from 4.6 to 5.0 $\mathrm{eV}[32,33,34]$ and the $\mathrm{EA}_{F 4 T C N Q}$ was reported from 5.08 to $5.24 \mathrm{eV}[35,36]$. Even though the IE of S-P3MEET was measured to be $\mathrm{IE}_{S-P 3 M E E T} \approx 5.1 \mathrm{eV}$ [28], a simple judgement of doping or non-doping cannot be made just based on these two numbers. Therefore, further experimental verification is still necessary in order to demonstrate the ability of F4TCNQ to dope S-P3MEET.

Near-edge X-ray absorption fine structure (NEXAFS) spectroscopy is a powerful technique to investigate the bonding and oxidation states of polymers and small molecules. Photoabsorption excites and ionizes 1s electrons, leaving empty core states. The resulting energy loss spectrum gives unique spectroscopic fingerprints that can be assigned to specific chemical groups. The spectrum for electronically delocalized states is difficult to calculate because it is very sensitive to intermolecular interactions like $\pi-\pi$ stacking and doping [37, 38]. The normalized carbon K-edge total electron yield (TEY) spectra of neat and $1 \mathrm{wt} \%$ F4TCNQ doped S-P3MEET samples are shown in Fig. 3. The spectrum of neat F4TCNQ is also plotted for reference. Detailed peak assignments of F4TCNQ are not shown here but can be found in published literature [23]. As can be seen, significant changes are observed between the doped and undoped S-P3MEET films. The peak at $285.2 \mathrm{eV}$, which corresponds to the $\mathrm{C} 1 \mathrm{~s}(\mathrm{C}=\mathrm{C}) \rightarrow \pi^{*}{ }_{C=C}$ transition and is associated with aromatic carbons in the thiophene rings, increases dramatically upon p-doping $[16,39,40]$. Note that no linear combination of the spectra from pure F4TCNQ and SP3MEET can account for the increased intensity of the pre-edge peak. The increased intensity of the pre-edge peak indicates an increased density of delocalized hole states in the S-P3MEET backbone, corresponding to p-type doping. Similar results have also been reported for the p-type doping of P3HT by PSS [13] and SP3MEET doped with a PFI, Nafion [16]. In addition, the peak around $293 \mathrm{eV}$ (associated with $\sigma^{*}{ }_{C-F}$ excitations [41, 42]) also increases in intensity as expected due to the presence of F4TCNQ. Finally, no significant changes are found with increased annealing temperature up to $180^{\circ} \mathrm{C}$ other than slightly increased intensity of the $\mathrm{C} 1 \mathrm{~s}(\mathrm{C}-\mathrm{H}) \rightarrow \sigma^{*}{ }_{C-H}$ transition peaks around $300 \mathrm{eV}$ (Supporting Information Fig. S1). Since TEY detected NEXAFS is a surface sensitive technique with electron escape depth $\sim 5 \mathrm{~nm}$ [43], this result indicates no change to surface composition. The increased intensity at $300 \mathrm{eV}$ is likely due to the subtle structural rearrangement of S-P3MEET with more side-chains at the film/air interface after annealing. Given that the spectra of doped films at mild and high heating are quite similar, it implies that unlike PFI doped S-P3MEET, where the doping process occurs only at elevated temperature [16], F4TCNQ doping occurs spontaneously.

The sharp increase of the near edge peak in the NEXAFS spectrum with addition of F4TCNQ is clear evidence that SP3MEET is p-type doped by F4TCNQ at low doping concentrations. However, NEXAFS is unable to either assess the efficiency of doping or predict how much the conductivity or morphology of S-P3MEET is affected by the presence of dopants. Therefore, further investigation on dopant induced conductivity is necessary. The in-plane conductivity $(\sigma)$ of S-P3MEET films with doping concentrations up to $10 \mathrm{wt} \%$ are shown in Fig. 4. The $\sigma$ of neat S-P3MEET film is measured to be $98.7 \pm 10.8$ $\mathrm{mS} / \mathrm{cm}$, which is consistent with the previously reported value

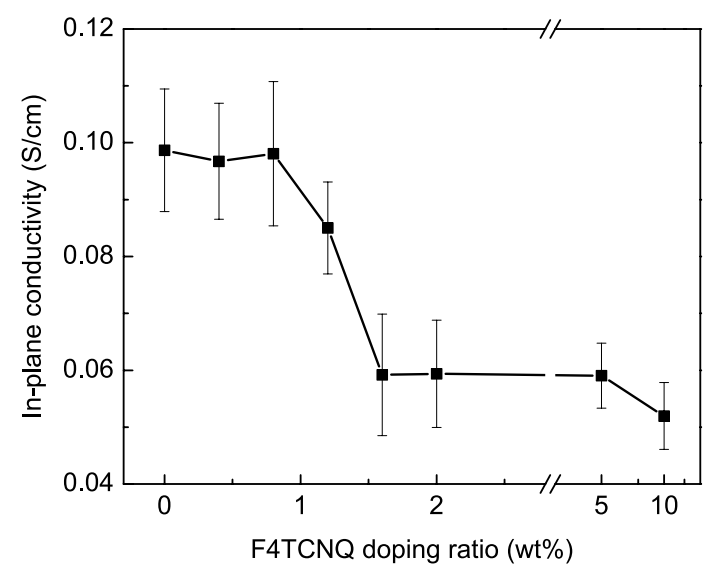

Figure 4: Conductivity measurements of S-P3MEET/F4TCNQ blend films as a function of F4TCNQ concentration. Conductivity was measured by a fourpoint probe method. 


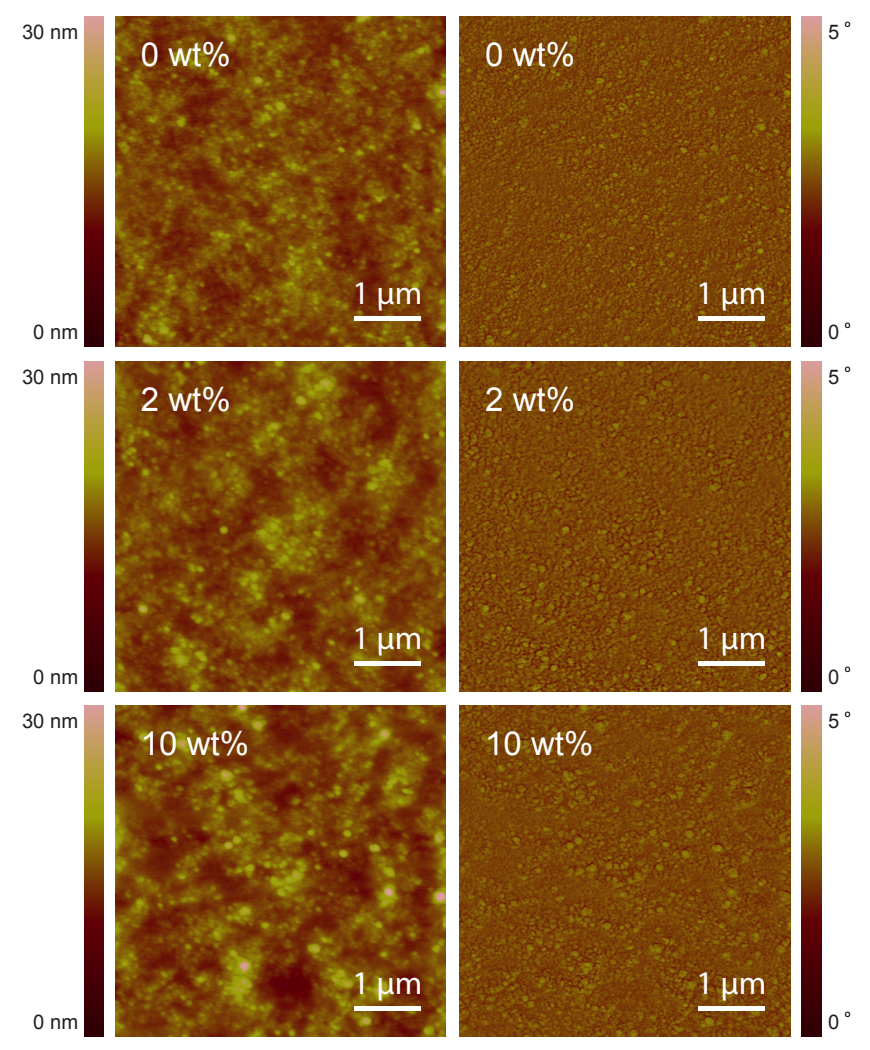

Figure 5: Topography (left) and phase (right) images of neat S-P3MEET and S-P3MEET/F4TCNQ blend films obtained from TM-AFM.

[16]. The blend films show a decrease in conductivity of 50$60 \%$ once the F4TCNQ concentration reaches $1.2 \mathrm{wt} \%$. Since molecular doping was expected to increase the conductivity of conjugated polymers $[44,45]$, the decreased conductivity beyond $1.2 \mathrm{wt} \%$ doping shown here was unexpected. One possible explanation for this effect is that F4TCNQ causes morphological changes in the film that result in reduced charge transport.

In order to investigate the effect of F4TCNQ doping on the S-P3MEET surface morphology, the films were examined using atomic force microscopy (AFM). The topography and phase images at representative doping concentrations are shown in Fig. 5. The full doping concentration series of images can be found in the Supporting Information (Fig. S2). Also, the root mean square (RMS) roughness values obtained from the topography and phase images are listed in Table 1. As can be seen, only subtle changes in topography and phase are observed upon doping. The topography roughness only increases slightly from $1.5 \mathrm{~nm}$ to $2.2 \mathrm{~nm}$ for a film with thickness $\sim 30 \mathrm{~nm}$, while the phase roughness remains the same. These results clearly prove that the conductivity decrease does not result from morphological changes to the S-P3MEET.

To further study the microstructural effect of adding F4TCNQ to S-P3MEET, the samples were examined using differential scanning calorimetry (DSC). The DSC curves for SP3MEET with 0-10 wt\% F4TCNQ are shown in Fig. 6. The neat S-P3MEET heating curve (black) shows a strong negative feature with onset at $98{ }^{\circ} \mathrm{C}$ that corresponds to the side-chain
Table 1: Topography $\left(\mathrm{r}_{z}\right)$ and phase $\left(\mathrm{r}_{p}\right)$ RMS roughness values from TM-AFM for neat S-P3MEET and S-P3MEET/F4TCNQ blend films

\begin{tabular}{ccc}
\hline $\begin{array}{c}\text { Doping Concentration } \\
(\boldsymbol{w} \%)\end{array}$ & $\begin{array}{c}\text { Topography Roughness } \boldsymbol{r}_{z} \\
(\mathbf{n m})\end{array}$ & $\begin{array}{c}\text { Phase Roughness } \boldsymbol{r}_{p} \\
(\mathbf{d e g})\end{array}$ \\
\hline 0 & 1.55 & 0.18 \\
0.5 & 1.46 & 0.17 \\
1 & 1.48 & 0.18 \\
2 & 1.68 & 0.16 \\
5 & 1.99 & 0.18 \\
10 & 2.16 & 0.21 \\
\hline
\end{tabular}

melting, and a broad weak negative feature ranging from $230^{\circ} \mathrm{C}$ to $270^{\circ} \mathrm{C}$ that is correlated to the backbone melting the of polymer. Normally, the melting temperature is lowered upon addition of an impurity to a pure compound due to increased entropy upon mixing [46]. However, that is not the case here. Addition of F4TCNQ to S-P3MEET results in a continuous increase in the side-chain melting temperature, which increases by $\sim 30{ }^{\circ} \mathrm{C}$ with addition of $5 \mathrm{wt} \%$ F4TCNQ.

The increased melting temperature of the side-chains with added F4TCNQ implies: (1) that the side-chains interact with the F4TCNQ and (2) that the presence of F4TCNQ stabilizes and/or orients the side-chains. The NEXAFS spectrum provides strong evidence that F4TCNQ is charged in S-P3MEET. The charged F4TCNQ causes the polar bonds in the side chains to orient due to the Coulombic interaction. This is a well known effect in crown ether [47] and poly(ethylene oxide) (PEO) [48] systems. It is therefore reasonable to assign the increased 


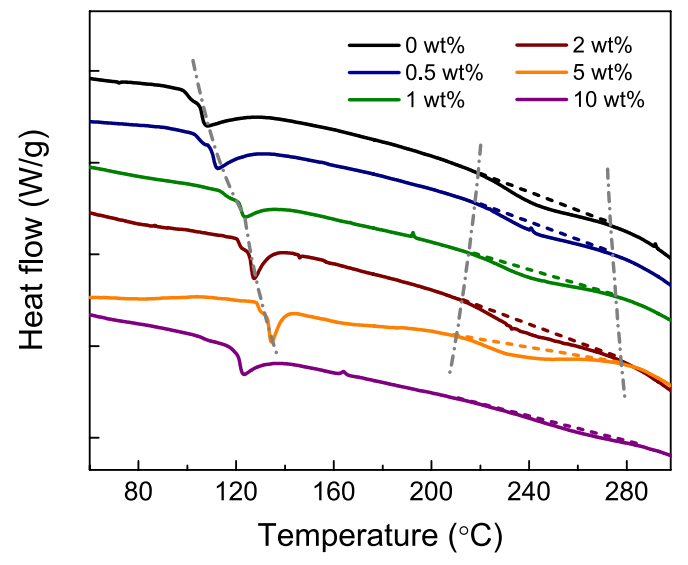

Figure 6: Heat flow vs. temperature measured using a DSC for samples of S-P3MEET doped with different F4TCNQ concentrations.

side-chain melting temperature to orientation of the polar $\mathrm{C}-$ $\mathrm{O}$ bonds around the charged F4TCNQ anion. We also note that the side-chain melting decreased slightly with $10 \mathrm{wt} \%$ doping, which shows that maximum side chain interaction with F4TCNQ is reached between 5 and $10 \mathrm{wt} \%$ F4TCNQ loading. Since the side chains could only orient to charged F4TCNQ, we posit that the maximum doping level is reach at between 5 and $10 \mathrm{wt} \%$ doping. In the following dopant diffusion studies, 10 wt\% F4TCNQ doping is chosen for maximum contrast.

To summarize, given the evidence of the increased charge density from NEXAFS experiment and the strong side-chains interaction from DSC experiment, it is reasonable to assume that the molecular dopant F4TCNQ undergoes either full charge separation or partial charge transfer with the S-P3MEET. Even though addition of F4TCNQ does not increase the conductivity, all other evidence points to charging of F4TCNQ. We do not assign a mechanism to the charging of F4TCNQ because there is no strong evidence for either partial of full charge transfer, nor is the determination of the mechanism central to this article. There is some evidence that the F4TCNQ charging can compete with the doping by the sulfonate groups on the S-P3MEET for counter ions. Beside the lack of conductivity increase is the thermal persistence of the NEXAFS data. Doped samples heated to $110^{\circ} \mathrm{C}$ and $180^{\circ} \mathrm{C}$ have identical spectra (Supporting Information Fig. S1), while F4TCNQ clearly leaves the P3HT layer at less than $150{ }^{\circ} \mathrm{C}$. Also the AFM phase images are unaltered upon doping. If there was no doping competition, we would expect F4TCNQ to remain neutral and to form separate domains, which would result in substantial roughening of the phase image. Finally, the melting temperature range for the backbone does not change much, but broadens slightly, indicating that there is only a very small change in the doping density along the polymer backbone, which again argues for a doping competition between F4TCNQ and the sulfonate groups. Significantly more spectroscopic work would need to be performed to assign this charging mechanism more accurately.

\subsection{Dopant Site Choice upon Thermal Annealing}

In the above section, we have shown that F4TCNQ is also able to dope S-P3MEET. However, in typical device architectures, other non-polar layers, such as P3HT, are stacked adjacent to the polar layer. We have also shown in section 3.1 that P3HT can be doped by F4TCNQ. The questions then arise whether the F4TCNQ has preference to stay in the polar layer (e.g. S-P3MEET) or in the non-polar layer (e.g. P3HT), and how thermal annealing affects this site choice.

The logic of the experiments in this section is that polar/nonpolar (S-P3MEET/P3HT) bilayers are fabricated. F4TCNQ starts either in the polar or the non-polar layer at room temperature and the adjacent layer starts free of dopant. Since SP3MEET is almost transparent and does not fluoresce, the diffusion of dopant upon thermal annealing is observed by monitoring the change of absorbance and/or fluorescence quenching of P3HT layer only.
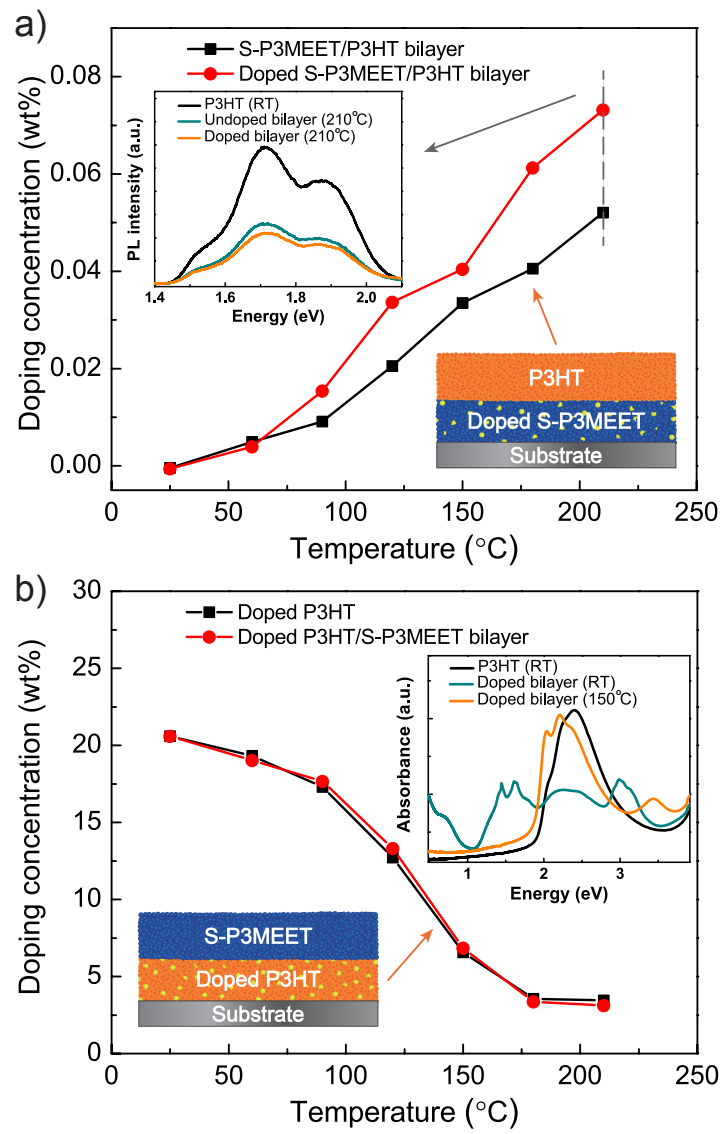

Figure 7: a) Doping concentration in P3HT film determined from photoluminescence quenching of undoped (black) and doped (red) S-P3MEET/P3HT bilayer samples at elevated temperatures. Inset cartoon: prepared bilayer structure. Inset figure: photoluminescence spectra of both undoped (cyan) and doped (orange) S-P3MEET/P3HT bilayer samples after annealing at $210{ }^{\circ} \mathrm{C}$. b) Doping concentration in $\mathrm{P} 3 \mathrm{HT}$ film determined from the ground-state/polaron peak ratio in UV-vis-NIR spectra of doped P3HT film (black) and doped P3HT/SP3MEET bilayer (red) at elevated temperatures. Inset cartoon: prepared bilayer structure. Inset figure: UV-vis-NIR spectra of doped P3HT/S-P3MEET bilayer sample before (cyan) and after (orange) annealing at $150{ }^{\circ} \mathrm{C}$. The spectrum of neat P3HT (black) is also shown for reference. 
First, the bilayer sample with dopant starting in the non-polar layer is investigated. A P3HT film was spin-coated onto a 10 wt\% F4TCNQ doped S-P3MEET layer (cartoon in Fig. 7a). An undoped bilayer sample was also prepared for reference. The UV-vis absorbance and photoluminescence (PL) spectra of both samples were then recorded before and after thermal annealing. The absorbance spectra of all samples (Supporting Information Fig. S3a) do not show any significant differences other than a slightly more pronounced vibrionic peak at $2.03 \mathrm{eV}$ due to re-crystallization of $\mathrm{P} 3 \mathrm{HT}$ with annealing $[49,50]$. If P3HT were oxidized by F4TCNQ or at the surface with S-P3MEET, the P3HT absorption would be bleached and polaron absorption peaks would be seen at $1.55 \mathrm{eV}$, as seen in Fig. 2 [51]. However, the F4TCNQ concentration in the P3HT film is not high enough to see these doping features in the absorbance spectrum at any temperature. Based on previous work with P3HT, this means that the doping level is below $0.6 \mathrm{wt} \%$ [31].

The P3HT photoluminescence, on the other hand, is more sensitive to the presence of impurities such as F4TCNQ because exciton diffusion allows for sensing over a much larger volume $[52,53,54]$. The inset of Fig. 7a shows partial quenching of the photoluminescence upon thermal annealing at $210{ }^{\circ} \mathrm{C}$, and the full temperature series of PL quenching results can be found in the Supporting Information Fig. S3b. Assuming that all PL quenching is the result of dopants diffusing into the P3HT layer, the doping concentration can be determined by comparison to a calibration curve [31]. The total extrinsic doping concentration in $\mathrm{P} 3 \mathrm{HT}$ as a function of annealing temperature is plotted in Fig. $7 \mathrm{a}$.

Upon thermal annealing, both doped and undoped bilayer samples show evidence of P3HT quenching. The increased PL quenching in the undoped bilayer sample (black line) after annealing could be caused by interfacial doping of the P3HT by the S-P3MEET sulfonate side groups [16, 17]. We assume that this type of doping occurs in both samples. However, the calculated doping level in P3HT in the doped S-P3MEET sample (red line) is still higher than for the undoped sample. We attribute the difference to the F4TCNQ diffusing from the SP3MEET layer into the P3HT and quenching the fluorescence. Considering that both doped and undoped samples show close to identical quenching after annealing, it implies that most of the PL quenching is caused by mixing of the P3HT with SP3MEET. F4TCNQ only dopes P3HT to $<0.03 \mathrm{wt} \%$ at any annealing temperature and is not the most important quenching agent in the annealed bilayers. In addition, the results from Fig. 2 in section 3.1 show that the efficient diffusion of F4TCNQ into the P3HT layer results in complete quenching of PL. However, the doped S-P3MEET/P3HT bilayer sample here still retain $40 \%$ photoluminescence even after heating up to $210{ }^{\circ} \mathrm{C}$. All these combined results imply that under thermal stress, either F4TCNQ still remains in S-P3MEET or if F4TCNQ is diffusing out of S-P3MEET, it does not stay in the P3HT layer but instead sublimates away.

Second, the bilayer sample with dopant starting in the polar layer is examined. A S-P3MEET film was spin-coated on top of $20.6 \mathrm{wt} \%$ (17 mol\%) F4TCNQ doped P3HT (cartoon in Fig. $7 b)$. The $20.6 \mathrm{wt} \%$ doping ratio is chosen because it is reported as the concentration at which the blend F4TCNQ/P3HT system achieves maximum conductivity [24]. Upon thermal annealing, the UV-vis spectra (the inset of Fig. 7b) show reduced P3HT absorbance around $2.48 \mathrm{eV}$ and the appearance of the broad peaks centered around $1.5 \mathrm{eV}$ and $0.6 \mathrm{eV}$ which are characteristics of $\mathrm{P}_{3} \mathrm{HT}^{+}$polarons as seen in Fig. 2 [24, 25]. Based on the ground-state/polaron peak ratio, we also quantify the doping concentration in the P3HT layer [31] and the results are plotted in Fig. 7b. Interestingly, the presence of a S-P3MEET top layer makes no difference on the removal behavior of F4TCNQ from P3HT. After annealing to $180{ }^{\circ} \mathrm{C}$ the F4TCNQ concentration is reduced from 20.6 to $3.7 \mathrm{wt} \%$. Since no spectroscopic evidence of neutral F4TCNQ appears, it is safe to conclude that the F4TCNQ leaves the P3HT layer. However, the question of whether F4TCNQ stays in S-P3MEET or sublimates out of the sample under thermal stress cannot be determined from these measurements.

Reflectometry techniques such as neutron reflectometry (NR) or X-ray reflectometry (XRR) have recently been used to measure and quantify the vertical concentration of impurities in mixtures of polymers with fullerenes or other small molecules $[55,56,38,57,23,58,59,60]$. The X-ray reflectivity curves for a bilayer of P3HT on top of S-P3MEET doped with F4TCNQ
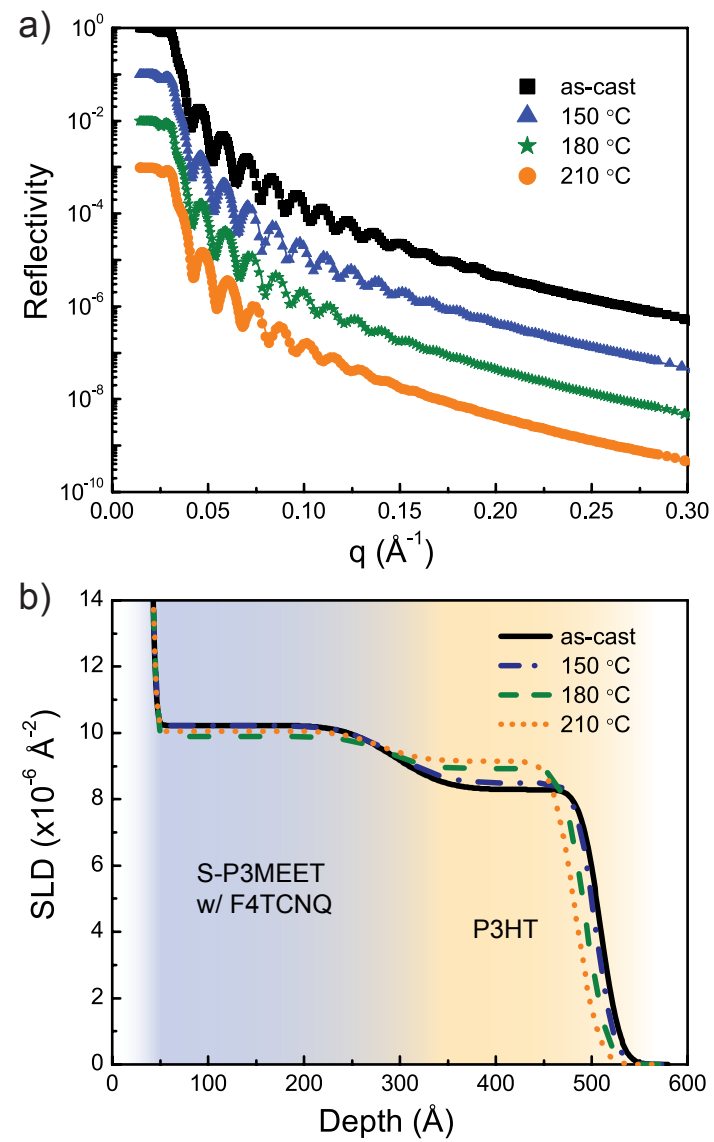

Figure 8: X-ray reflectometry curves along with model fits a), and corresponding scattering length density (SLD) profiles b) of a bilayer of $20 \mathrm{~nm}$ P3HT on top of $30 \mathrm{~nm} \mathrm{S-P3MEET} \mathrm{containing} 10 \mathrm{wt} \%$ F4TCNQ. Data were taken as-cast (black) and after heating to $150^{\circ} \mathrm{C}$ (navy), $180^{\circ} \mathrm{C}$ (green) and $210^{\circ} \mathrm{C}$ (orange). 
Table 2: X-ray reflectometry fitting parameters and calculation results for F4TCNQ doped S-P3MEET/P3HT bilayer sample.

\begin{tabular}{|c|c|c|c|c|c|c|c|c|c|c|c|}
\hline \multirow[b]{2}{*}{$\begin{array}{l}\text { Annealing } \\
\text { Temperature }\end{array}$} & \multicolumn{5}{|c|}{ S-P3MEET Layer } & \multicolumn{4}{|c|}{ P3HT Layer } & \multicolumn{2}{|c|}{ Total Bilayer } \\
\hline & $\begin{array}{c}S L D \\
\left(\times 10^{-6} \AA^{-2}\right)\end{array}$ & $\begin{array}{l}\text { Thickness } \\
(\AA)\end{array}$ & $\begin{array}{c}\text { Roughness } \\
(\AA)\end{array}$ & $\begin{array}{c}\text { S-P3MEET } \\
\text { Density }\left(\mathrm{g} / \mathrm{cm}^{3}\right)\end{array}$ & $\begin{array}{c}\text { F4TCNQ Doping } \\
\text { Fraction (\%) }\end{array}$ & $\begin{array}{c}S L D \\
\left(\times 10^{-6} \AA^{-2}\right)\end{array}$ & $\begin{array}{c}\text { Thickness } \\
(\AA)\end{array}$ & $\begin{array}{c}\text { Roughness } \\
(\AA)\end{array}$ & $\begin{array}{c}\text { P3HT Density } \\
\left(\mathrm{g} / \mathrm{cm}^{3}\right)\end{array}$ & $\begin{array}{c}\text { Thickness } \\
(\AA)\end{array}$ & $\begin{array}{c}\text { Mass Loss } \\
(\%)\end{array}$ \\
\hline As-cast & 10.23 & 253.2 & 42.0 & 0.98 & 29.5 & 8.29 & 213.9 & 16.3 & 0.90 & 467.1 & 0.0 \\
\hline $150^{\circ} \mathrm{C}$ & 10.22 & 252.2 & 41.8 & 1.00 & 29.0 & 8.49 & 209.1 & 16.1 & 0.92 & 461.3 & 0.1 \\
\hline $180^{\circ} \mathrm{C}$ & 9.90 & 244.3 & 34.6 & 0.98 & 14.5 & 8.93 & 206.6 & 17.9 & 0.97 & 450.9 & 2.1 \\
\hline $210^{\circ} \mathrm{C}$ & 10.01 & 240.7 & 35.3 & 0.99 & 19.1 & 9.15 & 199.9 & 18.4 & 0.99 & 440.6 & 2.5 \\
\hline
\end{tabular}

at elevated annealing temperatures are presented in Fig. 8a. From fitting reflectivity data for a neat P3HT layer, the scattering length density (SLD) of P3HT was determined to be $8.29 \times 10^{-6} \AA^{-2}$, which corresponds to a mass density of 0.9 $\mathrm{g} / \mathrm{cm}^{3}$ [61]. This value is slightly lower than reported from literature [62], but the measured density depends on purity, molecular weight, and sample preparation history. The SLD for F4TCNQ was calculated to be $11.8 \times 10^{-6} \AA^{-2}$ using a mass density value of $1.4 \mathrm{~g} / \mathrm{cm}^{3}$ [36]. Finally, we measured the SLD of S-P3MEET to be $9.59 \times 10^{-6} \AA^{-2}$ for a neat sample.

The fits to the reflectivity curves, based on a two-layer SLAB model, are presented in Fig. 8b. Depicted is the SLD vs. the vertical position in the film. The substrate of the sample is shown to the left of the plot at a depth of $0 \AA$ and the right of the plot is the surface. The plot shows that the S-P3MEET layer loses some SLD while the P3HT layer gains SLD with increased annealing temperature. In addition, the entire film stack loses some thickness ( 5\%). Quantitative analysis of the reflectometry data was performed to observe the dopant diffusion behavior. Several assumptions were made for the model:

1. The SLD of F4TCNQ is constant;

2. Movement of F4TCNQ will not affect the total volume of the film stack;

3. All mass loss is caused by evaporation of F4TCNQ;

4. No F4TCNQ stays in P3HT layer. The SLD change of P3HT layer only comes from P3HT density change.

Assumption 4 can be justified by the PL quenching results presented in Fig. 7a, where only less than 0.03 wt $\%$ F4TCNQ was found within the P3HT part of the bilayer structure after annealing to any temperature. In addition, If we assume that all of the change in SLD comes from movement of F4TCNQ, then we calculate increased F4TCNQ concentration in the P3HT layer with increased annealing temperature, which is the opposite to the result measured using PL measurements. Instead, if the density of P3HT and dopant concentration in S-P3MEET layer are allowed to change, we find a model consistent with the optical data. The details are as follows. First, after annealing to $180{ }^{\circ} \mathrm{C}$, the sample loses $\sim 2 \%$ of the total mass due to evaporation of $\sim 50 \%$ of the initial F4TCNQ. Typically, neat F4TCNQ is thermally evaporated using a $80{ }^{\circ} \mathrm{C}$ source in vacuum [63]. Since we saw no mass loss for annealing temperatures up to 150 ${ }^{\circ} \mathrm{C}$, we can safely conclude that F4TCNQ binds tightly enough in the S-P3MEET/P3HT bilayer to resist evaporation until the annealing temperature exceeds $150{ }^{\circ} \mathrm{C}$. Next, the S-P3MEET layer maintains a near constant density but loses $1.3 \mathrm{~nm}$ in thickness. This thickness loss is due to de-mixing with the $\mathrm{P} 3 \mathrm{HT}$ layer, as is seen in the reduction of the S-P3MEET/P3HT interface roughness. At the same time, heating to increasingly higher temperatures allows P3HT to re-crystallize and densify. We see increased density in $\mathrm{P} 3 \mathrm{HT}$ which is consistent with literature reports [64]. The fitting parameters and calculation results are given in Table 2.

An explanation of the data can be reached using both the spectroscopy and reflectometry data. The photoluminescence of P3HT is not quenched significantly by F4TCNQ from the S-P3MEET layer, even with thermal annealing. The XRR data fit shows that $\sim 50 \mathrm{wt} \%$ of the original F4TCNQ remains in the S-P3MEET, which corresponds to $\sim 5 \mathrm{wt} \%$ of the S-P3MEET. Recall from the DSC data in Fig. 6 that addition of $5 \mathrm{wt} \%$ F4TCNQ causes a strong increase in the side chain melting point of the S-P3MEET. It is therefore a plausible conclusion that orientation of the side chains around the dopant anions makes S-P3MEET much better at trapping F4TCNQ. But with $10 \mathrm{wt} \%$ doping, only one half of the F4TCNQ molecule are charged, and so the uncharged half sublimates from the sample. Although F4TCNQ can dope P3HT as well, the P3HT side chains are non-polar alkanes that are repelled from an anion and so, as shown in Fig. 7, the F4TCNQ sublimates out of P3HT at much lower temperatures. We must admit that a second explanation of the data could be that a chemical reaction between F4TCNQ and an electron rich group like $-\mathrm{SO}_{3}^{-}$could covalently bind the F4TCNQ to the polymer and thereby make the dopant insoluble. However, if this chemical reaction exists, we would expect to have seen its existence in the DSC data. With increased F4TCNQ concentration this reaction would have used or released more heat. But there is no peak in the DSC data that grows with F4TCNQ concentration. We are therefore reasonably confident that the polarity of the side chain is a critical element to fixing the position of the charged anion and that the charged dopant has a strong preference for the polar layer because of this.

\section{Conclusions}

In conclusion, the small molecule dopant, F4TCNQ, is shown to diffuse readily into the $\mathrm{P} 3 \mathrm{HT}$ at room temperature and efficiently dope this non-polar layer. Meanwhile, the increased intensity of the pre-edge peak in NEXAFS spectra unambiguously proves that polar S-P3MEET material can also be doped with the addition of F4TCNQ by showing the increased number of empty states in the S-P3MEET due to the molecular or charge transfer (CT) state doping. However, a test of conductivity vs. doping concentration in S-P3MEET shows an unexpected decrease, which is inconsistent with the clearly strong doping interaction. Morphology analysis using AFM reveals that this inconsistency does not result from morphological effects. Calorimetry measurements of F4TCNQ in S-P3MEET 
show a strong ordering interaction between F4TCNQ and sidechain of S-P3MEET, with $5 \mathrm{wt} \%$ doping increasing the sidechain melting temperature by $\sim 30{ }^{\circ} \mathrm{C}$.

A second aspect of doping investigated was the thermal stability of dopant site. Since the dopants are not covalently attached to the polymer, they are free to diffuse if the Coulombic, dipolar, and van der Waals binding forces are overcome. S-P3MEET presents a particular challenge to monitor dopant diffusion because it is self-doped, and so does not fluoresce. Bilayer experiments with $\mathrm{P} 3 \mathrm{HT}$ as the reporter layer reveals that doped F4TCNQ binds in S-P3MEET with little leaving the layer even with heating to $150{ }^{\circ} \mathrm{C}$; this is a considerable increase over the $80{ }^{\circ} \mathrm{C}$ sublimation temperature. In contrast, very little F4TCNQ remains in the P3HT layer after annealing at $80{ }^{\circ} \mathrm{C}$. It is likely that the side-chain ordering of the PEO sidechain of S-P3MEET causes the strong binding of the F4TCNQ. The alkane side-chain of P3HT does have polar bonds to orient and so is not able to contribute to binding F4TCNQ. These results suggest that adding ethyl-oxy side-chains to polymers is in general a good method for stabilizing the position of molecular dopants. Finally, reflectometry results show that $5 \mathrm{wt} \%$ of the F4TCNQ still remained in the S-P3MEET layer even with heating to $210{ }^{\circ} \mathrm{C}$. Developing a better understanding of the mechanism behind dopant site choice upon thermal annealing will help in the development and search for more stable doping methods and lead to organic electronic devices with improved lifetime and stability.

\section{Acknowledgement}

This research project was supported by the U.S. Department of Energy, Office of Science, Office of Basic Energy Sciences, Neutron scattering division under grant number DESC0010419. The NEXAFS work was performed under the auspices of the U.S. Department of Energy by Lawrence Livermore National Laboratory under Contract DE-AC52-07NA27344. We would like to thank Elke Arrenholz and Alpha N'Diaye from ALS and Michael Toney, Badri Shyam from SSRL for user support and training. We would also like to thank Plextronics for the donation of S-P3MEET.

\section{References}

[1] S. R. Forrest, The path to ubiquitous and low-cost organic electronic appliances on plastic, Nature 428 (6986) (2004) 911-918.

[2] F. C. Krebs, T. D. Nielsen, J. Fyenbo, M. Wadstrom, M. S. Pedersen, Manufacture, integration and demonstration of polymer solar cells in a lamp for the "lighting africa" initiative, Energy Environ. Sci. 3 (2010) $512-525$.

[3] Z. He, C. Zhong, S. Su, M. Xu, H. Wu, Y. Cao, Enhanced powerconversion efficiency in polymer solar cells using an inverted device structure, Nat. Photonics 6 (2012) 591-595.

[4] W. C. Lin, W. B. Wang, Y. C. Lin, B. Y. Yu, Y. Y. Chen, M. F. Hsu, J. H. Jou, J. J. Shyue, Migration of small molecules during the degradation of organic light-emitting diodes, Org. Electron. 10 (2009) 581-586.

[5] S. Duhm, I. Salzmann, B. Broker, H. Glowatzki, R. L. Johnson, N. Koch, Interdiffusion of molecular acceptors through organic layers to metal substrates mimics doping-related energy level shifts, Appl. Phys. Lett. 95 (2009) 093305.
[6] C. H. Peters, I. T. Sachs-Quintana, J. P. Kastrop, S. Beaupré, M. Leclerc, M. D. McGehee, High efficiency polymer solar cells with long operating lifetimes, Adv. Energy Mater. 1 (4) (2011) 491-494.

[7] M. Jørgensen, K. Norrman, S. A. Gevorgyan, T. Tromholt, B. Andreasen, F. C. Krebs, Stability of polymer solar cells, Adv. Mater. 24 (2012) 580612.

[8] L. Groenendaal, F. Jonas, D. Freitag, H. Pielartzik, J. R. Reynolds, Poly(3,4-ethylenedioxythiophene) and its derivatives: Past, present, and future, Adv. Mater. 12 (7) (2000) 481-494.

[9] S. Günes, H. Neugebauer, , N. S. Sariciftci, Conjugated polymer-based organic solar cells, Chem. Rev. 107 (4) (2007) 1324-1338.

[10] F. C. Krebs, Fabrication and processing of polymer solar cells: A review of printing and coating techniques, Sol. Energy Mater. Sol. Cells 93 (4) (2009) 394-412.

[11] H. Shi, C. Liu, Q. Jiang, J. Xu, Effective approaches to improve the electrical conductivity of pedot:pss: a review, Adv. Electron. Mater. 1 (4) (2015) 15000171500032.

[12] A. J. Moulé, K. Meerholz, Intensity-dependent photocurrent generation at the anode in bulk-heterojunction solar cells, Appl. Phys. B 92 (2) (2008) 209-218.

[13] D. M. Huang, S. A. Mauger, S. Friedrich, S. J. George, D. DumitriuLaGrange, S. Yoon, A. J. Moulé, The consequences of interface mixing on organic photovoltaic device characteristics, Adv. Funct. Mater. 21 (2011) 1657-1665.

[14] S. A. Mauger, L. Chang, C. W. Rochester, A. J. Moulé, Directional dependence of electron blocking in pedot:pss, Org. Electron. 13 (11) (2012) 2747-2756

[15] A. J. Moulé, M. C. Jung, C. W. Rochester, W. Tress, D. LaGrange, I. E. Jacobs, J. Li, S. A. Mauger, M. D. Rail, O. Lin, D. J. Bilski, Y. Qi, P. Stroeve, L. A. Berben, M. Reide, Mixed interlayers at the interface between pedot:pss and conjugated polymers provide charge transport control, J. Mater. Chem. C 3 (2015) 2664-2676.

[16] S. A. Mauger, J. Li, O. T. Ozmen, A. Y. Yang, S. Friedrich, M. D. Rail, L. A. Berben, A. J. Moulé, High work-function hole transport layers by self-assembly using a fluorinated additive, J. Mater. Chem. C 2 (2014) $115-123$.

[17] T. P. Le, Z. Shang, L. Wang, N. Li, S. V. Kesava, J. W. O'Connor, Y. Chang, C. Bae, C. Zhu, A. Hexemer, E. W. Gomez, A. Salleo, M. A. Hickner, E. D. Gomez, Miscibility and acid strength govern contact doping of organic photovoltaics with strong polyelectrolytes, Macromolecules 48 (15) (2015) 5162-5171.

[18] J. Blochwitz, M. Pfeiffer, T. Fritz, K. Leo, Low voltage organic light emitting diodes featuring doped phthalocyanine as hole transport material, Appl. Phys. Lett. 73 (6) (1998) 729-731.

[19] M. Pfeiffer, K. Leo, X. Zhou, J. Huang, M. Hofmann, A. Werner, J. Blochwitz-Nimoth, Doped organic semiconductors: Physics and application in light emitting diodes, Org. Electron. 4 (2-3) (2003) 89-103.

[20] K. Walzer, B. Maennig, M. Pfeiffer, K. Leo, Highly efficient organic devices based on electrically doped transport layers, Chem. Rev. 107 (4) (2007) 1233-1271.

[21] C. P. Liu, W. B. Wang, C. W. Lin, W. C. Lin, C. Y. Liu, C. H. Kuo, S. H. Lee, W. L. Kao, G. J. Yen, Y. W. You, H. Y. Chang, J. H. Jou, J. J. Shyue, Molecular migration behaviors in organic light-emitting diodes with different host structures, Org. Electron. 12 (2) (2011) 376-382.

[22] M. Hermenau, M. Riede, K. Leo, Stability and degradation of organic and polymer solar cells, John Wiley \& Sons, Ltd, 2012.

[23] J. Li, C. W. Rochester, I. E. Jacobs, S. Friedrich, P. Stroeve, M. Riede, A. J. Moule, Measurement of small molecular dopant f4tenq and c60f36 diffusion in organic bilayer architectures, ACS Appl. Mater. Interfaces.

[24] D. T. Duong, C. Wang, E. Antono, M. F. Toney, A. Salleo, The chemical and structural origin of efficient p-type doping in p3ht, Org. Electron. 14 (5) (2013) 1330-1336.

[25] P. Pingel, D. Neher, Comprehensive picture of p-type doping of p3ht with the molecular acceptor f4-tcnq, Phys. Rev. B 87 (2013) 115209.

[26] J. Gao, J. D. Roehling, Y. Li, H. Guo, A. J. Moule, J. K. Grey, The effect of 2,3,5,6-tetrafluoro-7,7,8,8-tetracyanoquinodimethane charge transfer dopants on the conformation and aggregation of poly(3-hexylthiophene), J. Mater. Chem. C 1 (36) (2013) 5638-5646.

[27] I. E. Jacobs, J. Li, S. L. Burg, D. J. Bilsky, B. T. Rotondo, M. P. Augustine, P. Stroeve, A. J. Moulé, Reversible optical control of conjugated polymer solubility with sub-micrometer resolution, ACS Nano 9 (2) (2015) 1905- 
1912.

[28] S. A. Mauger, A. J. Moulé, Characterization of new transparent organic electrode materials, Org. Electron. 12 (11) (2011) 1948-1956.

[29] A. Nelson, Co-refinement of multiple-contrast neutron/x-ray reflectivity data using motofit, J. Appl. Crystallogr. 39 (2) (2006) 273-276.

[30] E. E. Aziz, A. Vollmer, S. Eisebitt, W. Eberhardt, P. Pingel, D. Neher, N. Koch, Localized charge transfer in a molecularly doped conducting polymer, Adv. Mater. 19 (20) (2007) 3257-3260.

[31] J. Li, G. Zhang, D. M. Holm, I. E. Jacobs, B. Yin, P. Stroeve, M. Mascal, A. J. Moulé, Introducing solubility control for improved organic p-type dopants, Chem. Mater. 27 (16) (2015) 5765-5774.

[32] J. Frisch, A. Vollmer, J. Rabe, N. Koch, Ultrathin polythiophene films on an intrinsically conducting polymer electrode: Charge transfer induced valence states and interface dipoles, Org. Electron. 12 (6) (2011) 916922.

[33] I. W. Hwang, D. Moses, A. J. Heeger, Photoinduced carrier generation in p3ht/pcbm bulk heterojunction materials, J. Phys. Chem. C 112 (11) (2008) 4350-4354.

[34] S. Braun, W. R. Salaneck, M. Fahlman, Energy-level alignment at organic/metal and organic/organic interfaces, Adv. Mater. 21 (14-15) (2009) $1450-1472$

[35] K. Kanai, K. Akaike, K. Koyasu, K. Sakai, T. Nishi, Y. Kamizuru, T. Nishi, Y. Ouchi, K. Seki, Determination of electron affinity of electron accepting molecules, Appl. Phys. A 95 (1) (2009) 309-313.

[36] W. Gao, A. Kahn, Electronic structure and current injection in zinc phthalocyanine doped with tetrafluorotetracyanoquinodimethane: Interface versus bulk effects, Org. Electron. 3 (2) (2002) 53-63.

[37] B. Watts, S. Swaraj, D. Nordlund, J. Lüning, H. Ade, Calibrated nexafs spectra of common conjugated polymers, J. Chem. Phys. 134 (2) (2011) 024702 .

[38] S. A. Mauger, L. L. Chang, S. Friedrich, C. W. Rochester, D. M. Huang, P. Wang, A. J. Moulé, Self-assembly of selective interfaces in organic photovoltaics, Adv. Funct. Mater. 23 (15) (2013) 1935-1946.

[39] C. Lenardi, E. Barborini, V. Briois, L. Lucarelli, P. Piseri, P. Milani, \{NEXAFS \} characterization of nanostructured carbon thin-films exposed to hydrogen, Diamond Relat. Mater. 10 (3-7) (2001) 1195-1200.

[40] J. Stöhr, NEXAFS spectroscopy, Springer Science \& Business Media, 2013.

[41] M. Koshino, H. Kurata, S. Isoda, Dv-xa calculation of electron energy-loss near edge-structures of 2,3,5,6-tetrafluoro-7,7,8,8tetracyanoquinodimethane (f4tcnq), J. Electron. Spectrosc. Relat. Phenom. 135 (2-3) (2004) 191-200.

[42] D. Schmeisser, M. Tallarida, K. Henkel, K. Muller, D. Mandal, D. Chumakov, E. Zschech, Characterization of oxidic and organic materials with synchrotron radiation based xps and xas, Mater. Sci.-Poland 27 (1) (2009) 141-157.

[43] S. Anders, J. Daz, J. W. Ager, R. Yu Lo, D. B. Bogy, Thermal stability of amorphous hard carbon films produced by cathodic arc deposition, Appl. Phys. Lett. 71 (23) (1997) 3367-3369.

[44] B. Maennig, M. Pfeiffer, A. Nollau, X. Zhou, K. Leo, P. Simon, Controlled p-type doping of polycrystalline and amorphous organic layers: Self-consistent description of conductivity and field-effect mobility by a microscopic percolation model, Phys. Rev. B 64 (19) (2001) 195208.

[45] A. V. Tunc, A. De Sio, D. Riedel, F. Deschler, E. Da Como, J. Parisi, E. von Hauff, Molecular doping of low-bandgap-polymer:fullerene solar cells: Effects on transport and solar cells, Org. Electron. 13 (2) (2012) 290-296.

[46] D. R. Gaskell, Introduction to the thermodynamics of materials, Taylor and Francis Group, 2003.

[47] G. W. Gokel, W. M. Leevy, , M. E. Weber, Crown ethers: sensors for ions and molecular scaffolds for materials and biological models, Chem. Rev. 104 (5) (2004) 2723-2750.

[48] B. A. Deyerle, Y. Zhang, Effects of hofmeister anions on the aggregation behavior of peo-ppo-peo triblock copolymers, Langmuir 27 (15) (2011) 9203-9210

[49] J. D. Roehling, I. Arslan, A. J. Moule, Controlling microstructure in poly(3-hexylthiophene) nanofibers, J. Mater. Chem. 22 (2012) 24982506

[50] R. Noriega, J. Rivnay, K. Vandewal, F. P. V. Koch, N. Stingelin, P. Smith, M. F. Toney, A. Salleo, A general relationship between disorder, aggregation and charge transport in conjugated polymers, Nat. Mater. 12 (11)
(2013) 1038-1044.

[51] J. Guo, H. Ohkita, H. Benten, S. Ito, Near-ir femtosecond transient absorption spectroscopy of ultrafast polaron and triplet exciton formation in polythiophene films with different regioregularities, J. Am. Chem. Soc. 131 (46) (2009) 16869-16880.

[52] C. Williams, S. Lee, J. Ferraris, A. A. Zakhidov, Exciton-dopant and exciton-charge interactions in electronically doped oleds, J. Lumin. 110 (4) (2004) 396-406.

[53] K. H. Yim, G. L. Whiting, C. E. Murphy, J. J. M. Halls, J. H. Burroughes, R. H. Friend, J. S. Kim, Controlling electrical properties of conjugated polymers via a solution-based p-type doping, Adv. Mater. 20 (17) (2008) 3319-3324.

[54] W. C. Tsoi, S. J. Spencer, L. Yang, A. M. Ballantyne, P. G. Nicholson, A. Turnbull, A. G. Shard, C. E. Murphy, D. D. C. Bradley, J. Nelson, J.-S. Kim, Effect of crystallization on the electronic energy levels and thin film morphology of p3ht:pcbm blends, Macromolecules 44 (8) (2011) 2944 2952.

[55] K. H. Lee, P. E. Schwenn, A. R. G. Smith, H. Cavaye, P. E. Shaw, M. James, K. B. Krueger, I. R. Gentle, P. Meredith, P. L. Burn, Morphology of all-solution-processed "bilayer" organic solar cells, Adv. Mater. 23 (6) (2011) 766-770.

[56] J. W. Kiel, M. E. Mackay, B. J. Kirby, B. B. Maranville, C. F. Majkrzak, Phase-sensitive neutron reflectometry measurements applied in the study of photovoltaic films, J. Chem. Phys. 133 (7).

[57] J. D. Roehling, C. W. Rochester, H. W. Ro, P. Wang, J. Majewski, I. Batenburg, K. J.and Arslan, D. M. Delongchamp, A. J. Moulé, Material profile influences in bulk-heterojunctions, J. Polym. Sci., Part B: Polym. Phys. 52 (2014) 1291-1300.

[58] H. Chen, R. Hegde, J. Browning, M. D. Dadmun, The miscibility and depth profile of pcbm in $\mathrm{p} 3 \mathrm{ht}$ : thermodynamic information to improve organic photovoltaics, Phys. Chem. Chem. Phys. 14 (2012) 5635-5641.

[59] J. W. Kiel, B. J. Kirby, C. F. Majkrzak, B. B. Maranville, M. E. Mackay, Nanoparticle concentration profile in polymer-based solar cells, Soft Matter 6 (2010) 641-646.

[60] K. H. Lee, P. E. Schwenn, A. R. G. Smith, H. Cavaye, P. E. Shaw, M. James, K. B. Krueger, I. R. Gentle, P. Meredith, P. L. Burn, Morphology of all-solution-processed "bilayer" organic solar cells, Advanced Materials 23 (6) (2011) 766-770.

[61] National institute of standards and technology (nist) center for neutron research, scattering length density calculator [online]

[62] T. J. Prosa, M. J. Winokur, J. Moulton, P. Smith, A. J. Heeger, X-ray structural studies of poly(3-alkylthiophenes): an example of an inverse comb, Macromolecules 25 (17) (1992) 4364-4372.

[63] I. Bruder, S. Watanabe, J. Q. Qu, I. B. Muller, R. Kopecek, J. Hwang, J. Weis, N. Langer, A novel p-dopant with low diffusion tendency and its application to organic light-emitting diodes, Org. Electron. 11 (4) (2010) 589-593.

[64] T. Agostinelli, S. Lilliu, J. G. Labram, M. Campoy Quiles, M. Hampton, E. Pires, J. Rawle, O. Bikondoa, D. D. C. Bradley, T. D. Anthopoulos, J. Nelson, J. E. Macdonald, Real-time investigation of crystallization and phase-segregation dynamics in p3ht:pcbm solar cells during thermal annealing, Adv. Funct. Mater. 21 (9) (2011) 1701-1708. 
7. Graphical abstract

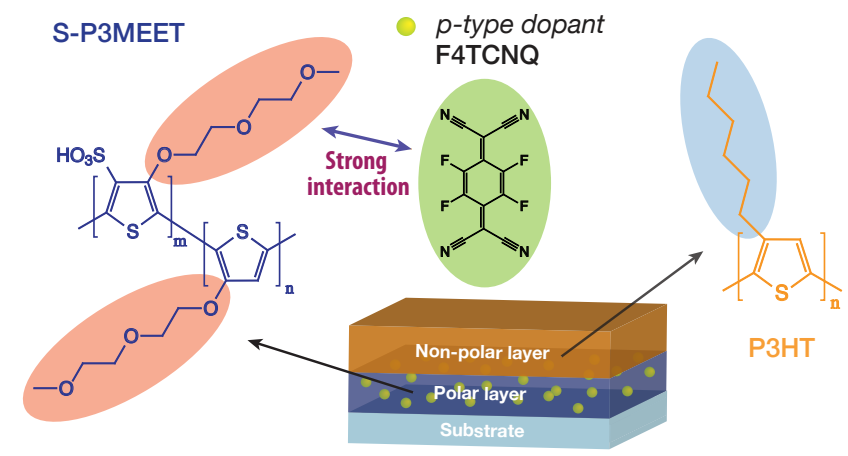

